The University of Southern Mississippi

The Aquila Digital Community

Faculty Publications

$5-1-2007$

\title{
Model-Independent Measurement of the Excited Fraction In a Magneto-Optical Trap
}

\author{
M. H. Shah \\ Kansas State University \\ H. A. Camp \\ Kansas State University \\ M. L. Trachy \\ Kansas State University \\ G. Veshapidze \\ Kansas State University \\ M.A. Gearba \\ University of Southern Mississippi
}

See next page for additional authors

Follow this and additional works at: https://aquila.usm.edu/fac_pubs

Part of the Physics Commons

\section{Recommended Citation}

Shah, M., Camp, H., Trachy, M., Veshapidze, G., Gearba, M., DePaola, B. (2007). Model-Independent Measurement of the Excited Fraction In a Magneto-Optical Trap. Physical Review A, 75(5).

Available at: https://aquila.usm.edu/fac_pubs/2019

This Article is brought to you for free and open access by The Aquila Digital Community. It has been accepted for inclusion in Faculty Publications by an authorized administrator of The Aquila Digital Community. For more information, please contact Joshua.Cromwell@usm.edu. 


\section{Authors}

M. H. Shah, H. A. Camp, M. L. Trachy, G. Veshapidze, M.A. Gearba, and B.D. DePaola 


\title{
Model-independent measurement of the excited fraction in a magneto-optical trap
}

\author{
M. H. Shah, ${ }^{1}$ H. A. Camp,,${ }^{1,2}$ M. L. Trachy, ${ }^{1}$ G. Veshapidze, ${ }^{1}$ M. A. Gearba,${ }^{3}$ and B. D. DePaola ${ }^{1}, *$ \\ ${ }^{1}$ J. R. Macdonald Laboratory, Department of Physics, Kansas State University, Manhattan, Kansas 66506-2601, USA \\ ${ }^{2}$ Institute for Defense Analyses, Science and Technology Division, 4850 Mark Center Drive, Alexandria, VA 22311-1882, USA \\ ${ }^{3}$ University of Southern Mississippi, Hattiesburg, Mississippi 39406, USA
}

(Received 9 June 2006; revised manuscript received 3 March 2007; published 31 May 2007)

\begin{abstract}
In many experiments involving a magneto-optical trap (MOT) it is of great importance to know the fraction of atoms placed in an excited state due to the trapping process. Generally speaking, researchers have had to use overly simplistic and untested models to estimate this fraction. In this work, the excited fractions of ${ }^{87} \mathrm{Rb}$ atoms in a MOT are directly measured using a charge transfer technique, for a range of MOT parameters. Simple models are then fit to the measured fractions. Using the results of this work, the excited fraction of ${ }^{87} \mathrm{Rb}$ atoms trapped in a MOT can be accurately estimated with knowledge of only the trapping laser intensity and detuning. The results are, at most, only weakly dependent on other MOT parameters.
\end{abstract}

DOI: 10.1103/PhysRevA.75.053418

PACS number(s): 32.80.Pj

\section{INTRODUCTION}

In many experiments involving atoms in a magnetooptical trap (MOT), it is important to know the spatial- and time-averaged fraction, $f$, of atoms put into an excited state due to the trapping process. For example, the most common method for estimating the total number of atoms in a MOT is to measure the fluorescence from the MOT, which leads to an estimate of the number of atoms in an excited state. This number, when divided by $f$, then yields the total number of atoms in the MOT. As another example, in cold collisions experiments, knowledge of the fraction of atoms in an excited state can be critical. However, most estimates of excited fractions are based on a simple model [1] for a twolevel system excited by an optical traveling wave, having a well-defined polarization, and incident on the MOT from a single direction:

$$
f=\frac{I / I_{s}}{1+2 I / I_{s}+(2 \delta / \Gamma)^{2}} .
$$

Here, $\delta$ is the detuning of the trapping laser frequency from resonance, $\Gamma$ is the full linewidth of the transition; for $\operatorname{Rb}\left(5 s_{1 / 2} \rightarrow 5 p_{3 / 2}\right), \Gamma / 2 \pi=5.98 \mathrm{MHz}$. Also, $I$ is the measured total trapping laser intensity, and $I_{s}$ is the so-called saturation intensity, often given [2] by

$$
I_{s}=\frac{2 \pi h c \Gamma}{3 \lambda^{3}} .
$$

In this expression, $\lambda$ is the transition wavelength, $c$ is the speed of light, and $h$ is Planck's constant. For the $\mathrm{Rb} D 2$ transition, Eq. (2) gives $I_{s}=3.28 \mathrm{~mW} / \mathrm{cm}^{2}$.

Equation (1) can also be written in terms of the saturation parameter, $s$ :

$$
f=\frac{s}{1+2 s},
$$

where

*Corresponding author. E-mail address: depaola@phys.ksu.edu

$$
s=\frac{I / I_{s}}{1+(2 \delta / \Gamma)^{2}} .
$$

A MOT is a much more complicated environment than the simple model of Eq. (1) was intended to describe. For example, the system under study in this work, trapped ${ }^{87} \mathrm{Rb}$, consists of more than two levels: there are two hyperfinesplit ${ }^{2} S_{1 / 2}$ ground states and four hyperfine-split excited states in the $5^{2} P_{3 / 2}$ manifold. Even more importantly, each of these hyperfine levels is Zeeman split by the MOT's magnetic field. There are three pairs of counter propagating beams of light, having different polarizations. This leads to complicated spatially and temporally varying optical intensities. Furthermore, optical pumping by these complicated fields yields an unknown distribution of $m_{F}$, which means that the nominal value of $I_{s}$ given in Eq. (2) could be inappropriate. Most importantly, the applicability of this model to a MOT has never been experimentally verified.

In measurements of the absolute cross sections for the photoionization of the $5 p$ state in $\mathrm{Rb}$, Dinneen et al. varied the trapping laser intensity while holding the detuning constant and measured the photoionization rate [3]. By fitting the ionization rate to an excited state saturation curve based on Eq. (1), and applying an overall scaling factor to ensure that $f$ saturated at a value of 0.5 , Dinneen et al. deduced an effective saturation intensity, $I_{s}$. They thereby obtained a model-dependent determination of $f$.

Recognizing that Eq. (1) represented a vast oversimplification of reality, Townsend et al. [4] proposed a slightly less severe approximation:

$$
f=\frac{C_{1}^{2} I / I_{s}}{1+2 C_{2}^{2} I / I_{s}+(2 \delta / \Gamma)^{2}},
$$

where $C_{1}$ and $C_{2}$ are average Clebsch-Gordon (CG) coefficients.

In a more rigorous effort, Javanainen [5] modeled the population dynamics in a MOT and fit his calculated populations to the ansatz: 


$$
\begin{gathered}
y=\left[\left(\frac{1}{\alpha}-\frac{1}{\beta}\right) \frac{s_{r}}{\left(s+s_{r}\right)}+\frac{1}{\beta}\right] s, \\
f=\frac{y}{1+2 y},
\end{gathered}
$$

where $\alpha, \beta$, and $s_{r}$ are the fitted parameters. Here, $\alpha^{-1 / 2}$ and $\beta^{-1 / 2}$ are effective CG coefficients at low and high values of $s$, respectively. The parameter $s_{r}$ is used to define what one means by "high" and "low." Note that for $s \ll s_{r}, f \rightarrow s / \alpha$, and for $s \gg s_{r}, f \rightarrow s / \beta$. Thus, for extreme values of the saturation parameter, Eqs. (6) become equivalent to Eq. (1), but with an effective value of saturation intensity given by $\widetilde{I}_{s}$ $=\alpha I_{s}$ (or $\left.\beta I_{s}\right)$. The result of Javanainen's work was recommended values of the three fitted parameters, which were found to depend on the total angular quantum numbers $F$ and $F^{\prime}$, the lower and upper levels of the trapping transition. As in the case of the other, simpler models, there has been no experimental verification of Javanainen's model.

So, the questions are, can the complicated physics in a MOT, as it pertains to the excited state fraction, be simply and conveniently modeled. And if so, how can the model be tested.

One answer to the second question is to use magnetooptical trap recoil ion momentum spectroscopy (MOTRIMS) $[6,7]$ to measure charge transfer between a beam of ions and the trapped atoms, as a probe of the excited state fraction. MOTRIMS is a well-established methodology that can be used to measure excited state fractions in a modelindependent manner. It is the intention of this paper to answer the first question for the special case of ${ }^{87} \mathrm{Rb}$, trapped on the ${ }^{2} S_{1 / 2}, F=2 \rightarrow{ }^{2} S_{3 / 2}, F^{\prime}=3$ cycling transition.

The idea, then, is to measure the fraction, $f$, as a function of $\delta$ and $I$. The next step is to compare the measurement to the three simple models already mentioned. We refer to these as the "one-parameter," "two-parameter," and "threeparameter" models, corresponding, respectively, to Eqs. (1), (5), and (6). The fitted parameters in these three models are an effective $I_{s}$ for the first, $C_{1}$ and $C_{2}$ for the second, and $\alpha$, $\beta$, and $s_{r}$ for the third.

\section{EXPERIMENT}

Details of the MOTRIMS methodology can be found in the literature [8], so only a brief description will be given here. When a target atom or molecule undergoes a charge transfer event with a projectile ion, a momentum kick is given to the target, which is then referred to as a "recoil ion." The momentum component $p_{\|}$that lies along the collision axis is related to energy defect, or $Q$ value of the collision by

$$
Q=-v_{p} p_{\|}-m_{e} v_{p}^{2} / 2
$$

where $v_{p}$ is the incoming projectile velocity and $m_{e}$ is the mass of an electron. The $Q$ value can be defined as the initial binding energy of the transferred electron on the target, minus the final binding energy of that electron on the projectile. Following charge transfer, the recoil ion is extracted from the interaction region by a weak $(\sim 10 \mathrm{~V} / \mathrm{cm})$ electric field, and

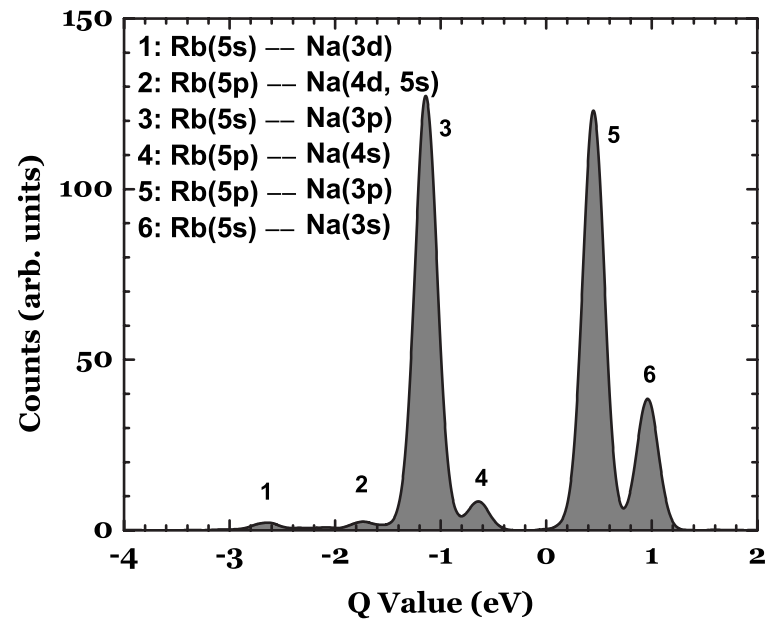

FIG. 1. Typical $Q$-value spectrum from which the excited fraction is determined. The peak labeled 5 is due to capture from $\mathrm{Rb}(5 p)$ to $\mathrm{Na}(3 p)$, while the peak labeled 6 is due to capture from $\mathrm{Rb}(5 s)$ to $\mathrm{Na}(3 s)$. These two peaks were used to measure the excited fraction in the MOT.

its recoil momentum vector is determined through the use of time of flight and two-dimensional position-sensitive detection. By plotting the charge transfer count rate as a function of measured $p_{\|}$, one can deduce the relative contributions of different charge transfer channels to the overall charge transfer cross section. A typical "Q-value spectrum" for $7 \mathrm{keV}$ $\mathrm{Na}^{+}$on trapped $\mathrm{Rb}$ is shown in Fig. 1. Each peak in the spectrum corresponds to a charge transfer channel. The number of counts in each of these peaks is proportional to the number of targets in the initial state times the charge transfer cross section for that channel. Thus, the relative count rates for each of the different channels, divided by the corresponding relative cross sections, directly give [7] the relative numbers of targets in the different initial states. That is, the excited state fraction is given by

$$
f=\frac{A_{p} / \sigma_{p}}{A_{p} / \sigma_{p}+A_{s} / \sigma_{s}}=\frac{A_{p}}{A_{p}+R A_{s}},
$$

where $A_{s}$ and $A_{p}$ are the areas under the peaks in the $Q$-value spectra corresponding to capture from $\mathrm{Rb}(5 s)$ and $\mathrm{Rb}(5 p)$, to $\mathrm{Na}(3 s)$ and $\mathrm{Na}(3 p)$, respectively, as shown in Fig. 1. $R$ $\equiv \sigma_{p} / \sigma_{s}$ is the ratio of cross sections for charge transfer for these channels. The relevant ratio of charge transfer cross sections has already been measured [9] for $7 \mathrm{keV} \mathrm{Na}^{+}$projectile ions to be $11.29 \pm 0.66$. Therefore, the relative number of counts in the charge transfer channels corresponding to capture from the $5 s$ and $5 p$ states of $\mathrm{Rb}$, measured as functions of trapping laser detuning and intensity, can be directly converted into a measure of the average excited state fraction for those trapping laser parameters.

Because the accuracy of the excited fraction reported here depends on the accuracy with which $R \equiv \sigma_{p} / \sigma_{s}$ was measured, it is appropriate to briefly describe that measurement. The essential idea is that if the trapping laser is turned off for a time that is long compared to the decay time of the $\mathrm{Rb}\left(5 p_{3 / 2}\right)$ state, but short compared to the time it takes the 
trapped atoms to move out of the path of the $\mathrm{Na}^{+}$beam, then the total number of atoms "seen" by the ion beam will not change, but the excited fraction will have gone to 0. (Here the laser-off time was $200 \mu \mathrm{sec}$.) That is,

$$
n_{s}+n_{p}=\text { const } \Rightarrow \Delta n_{s}+\Delta n_{p}=0
$$

where $\Delta n_{x}$ means the difference in the population of the $x$ th state with laser on and off. Furthermore, the area under any channel's peak in a $Q$-value spectrum, say the channel for charge transfer from $\mathrm{Rb}(x)$ to $\mathrm{Na}(y)$, is

$$
A_{x \rightarrow y} \propto n_{x} \sigma_{x y} \Rightarrow \Delta A_{x \rightarrow y} \propto \Delta n_{x} \sigma_{x y},
$$

where $\sigma_{x y}$ is the cross section for charge transfer from $\mathrm{Rb}(x)$ to $\mathrm{Na}(y)$, and the constant of proportionality contains beam overlap with target, detector efficiency, etc.

Then, taking the ratio of Eq. (10) for two channels, one involving capture from $\mathrm{Rb}(5 p)$ to $\mathrm{Na}(x)$, and the other from $\mathrm{Rb}(5 s)$ to $\mathrm{Na}(y)$, one obtains

$$
\frac{\Delta A_{p \rightarrow x}}{\Delta A_{s \rightarrow y}}=\frac{\sigma_{p \rightarrow x}}{\sigma_{s \rightarrow y}} \frac{\Delta n_{p}}{\Delta n_{s}}=-\frac{\sigma_{p \rightarrow x}}{\sigma_{s \rightarrow y}} \equiv-R,
$$

where the final equality is obtained using Eq. (9). Note that by taking the data in this way no prior knowledge of populations is required. Also note that any pair of channels corresponding to capture from the $\mathrm{Rb}(5 s)$ and $\mathrm{Rb}(5 p)$ states can be used, regardless of the final state in $\mathrm{Na}$ to which the electron is captured. For this work, the two rightmost channels shown in Fig. 1 were used. The right-most peak corresponds to $\mathrm{Rb}(5 s) \rightarrow \mathrm{Na}(3 s)$, while the next peak to the left corresponds to $\mathrm{Rb}(5 p) \rightarrow \mathrm{Na}(3 p)$. Finally, note that once the ratio of cross sections has been measured, one can determine the excited fraction using Eq. (8) without chopping the trapping lasers.

A standard optical setup, was used to cool and trap the ${ }^{87} \mathrm{Rb}$ atoms. An extended cavity diode laser was peak locked to the crossover peak midway between the $F=2 \rightarrow F^{\prime}=3$ and $F=2 \rightarrow F^{\prime}=2$ transitions in a saturated absorption spectrum, using a Zeeman-dithering scheme [10]. The output of the trap laser was then amplified to about $350 \mathrm{~mW}$ using a tapered amplifier [11].

The repump laser was locked to the $F=1 \rightarrow F^{\prime}=2$ peak in a second saturated absorption spectrum. In this case, the laser frequency was dithered around the transition, and no tapered amplifier was used on the output of the laser. The intensities of each of the trapping beams were measured using a power meter and scanning knife-edge beam profiler [12].

The MOTRIMS chamber has been described elsewhere in detail [8]. A source of $7 \mathrm{keV} \mathrm{Na}^{+}$ions was positioned on one side of the chamber, and two-dimensional position-sensitive recoil and projectile detectors (PSDs) were placed on the opposite side. An electrostatic deflector, located on the detector side of the chamber, steers projectile ions that have not undergone charge transfer, into a Faraday cup. The $7 \mathrm{keV} \mathrm{Na}$ atoms, neutral due to charge transfer from the $\mathrm{Rb}$, are undeflected and strike the "projectile PSD." The $\mathrm{Rb}^{+}$ions created in charge transfer events were extracted by a $10 \mathrm{~V} / \mathrm{cm}$ electric field, and directed onto the "recoil PSD." The flight time difference between the projectile and recoil ions gives $p_{\|}$, and

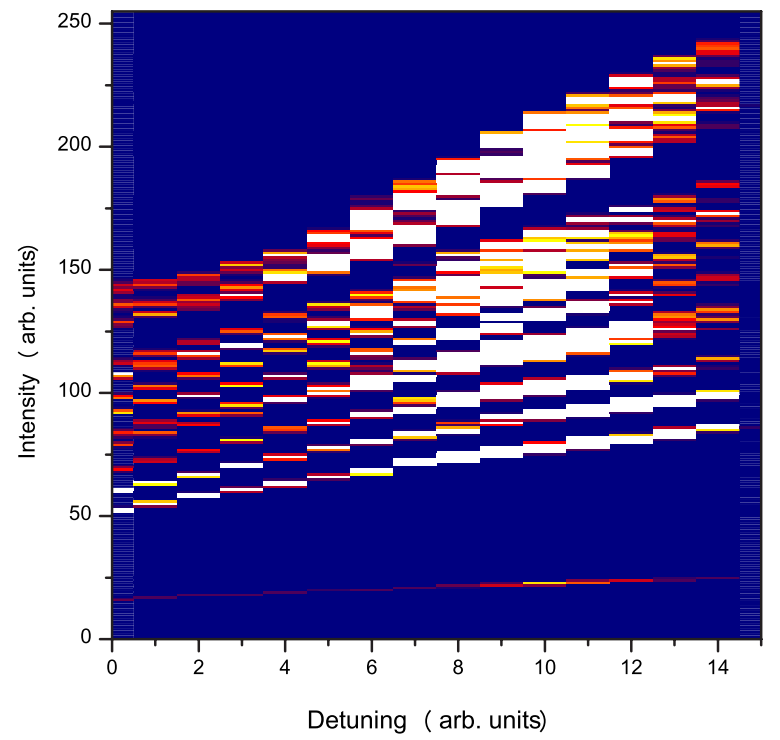

FIG. 2. (Color online) Measured excited fraction as a function of detuning (horizontal axis, arbitrary units) and trap laser intensity (vertical axis, arbitrary units). The measured fraction is shown in gray scale. The curvature in the plots reflects the dependence of the AOM efficiency on the applied radio frequency.

from this the $Q$ value with the aid of Eq. (7). Recoil ion counts are recorded as a function of $Q$ value, leading to "spectra" such as the one shown in Fig. 1.

The trapping laser was double passed through an acoustooptical modulator (AOM), and the value of $\delta$ was stepped by varying the input frequency to the AOM with a "staircase voltage generator." In this way, the detuning of the trapping laser from resonance was varied over the range $4.9 \geq \delta / \Gamma$ $\geq 1.57$. A sample of the staircase generator output was sent to an analog to digital converter (ADC). In general, the efficiency of the AOM is known to depend on the input frequency. Therefore, a measured fraction of the trapping laser beam was picked off downstream from the AOM and sent to an optical power meter, the output of which was sent to a second channel of the same ADC unit. Data were taken in event mode. That is, when a charge transfer event was detected, values of other parameters, such as laser power, detuning, and $\mathrm{Rb}$ ion positions and flight times were measured. Data were taken for several fixed (except for the aforementioned AOM efficiency effect) values of the trapping laser intensity. Following this procedure, $Q$-value spectra such as that shown in Fig. 1 were obtained for each detuning and trap laser intensity. The excited state fractions for each of these spectra were then determined using Eq. (8). Some of the analyzed data are shown in Fig. 2, which is a contour plot with detuning (in relative units) on the $x$ axis, trap laser intensity (in relative units) on the $y$ axis, and excited fraction in gray scale (false color in the online version).

\section{RESULTS}

Plots such as that shown in Fig. 2 can show overall trends, but are not useful vehicles for fitting multiparameter models to the measured data. Rather, it is convenient to plot the 


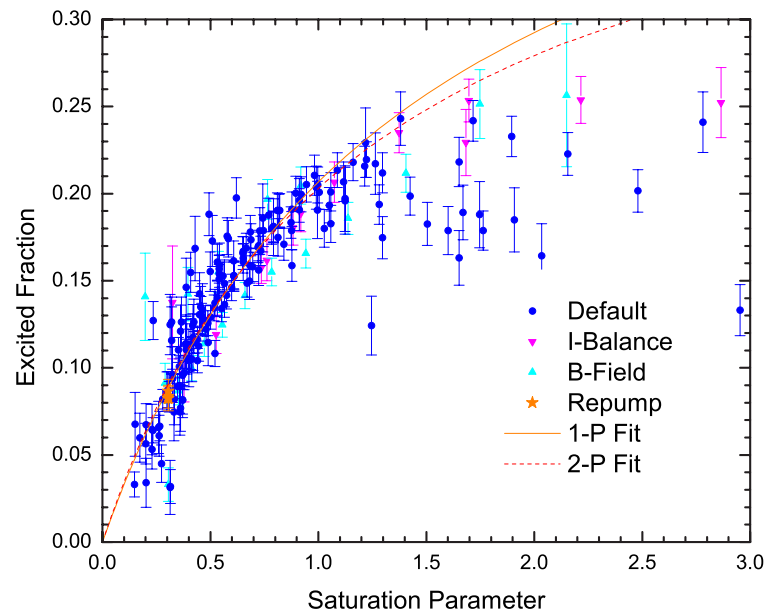

FIG. 3. (Color online) Excited state fraction versus the saturation parameter. The circles are data taken for default values of $B$-field gradient and trap laser balance. Data taken with other $B$-field gradients are shown as upward pointing triangles, while data taken for various balance between the different trapping laser beams are shown as downward pointing triangles. The stars are data for which the repump laser intensity has been varied. The solid and dashed curves are fits to the one- and two-parameter models described in the text.

measured $f$ versus the single variable $s$ from Eq. (4), using the "standard" definition [13] of $I_{s}$ from Eq. (2). The results of the measurements are shown as solid circles in the parametric plot of Fig. 3.

The error bars represent one standard deviation and result from counting statistics in the measurements of $A_{s}$ and $A_{p}$ from the $Q$-value spectra, and in uncertainty in the ratio of the collision cross sections, $R$. Using standard error analysis procedures, the estimated uncertainty in the measured fraction, designated by $f_{m}$ is given by

$$
\frac{\Delta f_{m}}{f_{m}}=\left(1-f_{m}\right)\left\{\left(\frac{\Delta A_{s}}{A_{s}}\right)^{2}+\left(\frac{\Delta A_{p}}{A_{p}}\right)^{2}+\left(\frac{\Delta R}{R}\right)^{2}\right\}^{1 / 2} .
$$

While there is some contribution to the measured excited fraction measurement due to $\Delta R$, the uncertainty is dominated by the statistical error and background subtraction in the individual $Q$-value spectra, taken for each value of $I$ and $\delta$.

The results of this work would be of minimal utility to the rest of the cold atom community if the excited state fraction was found to vary with other MOT parameters such as $B$-field gradient, balance in the six trapping beam intensities, or repump laser intensity. To test this dependence, these quantities were varied and additional measurements of the excited fraction were made. For data already described, the $B$-field gradient was $12.5 \mathrm{G} / \mathrm{cm}$, the intensities of the three pairs of trapping laser beams were in the ratio of 25.3:36.1:30.7, and the repump laser power was approximately $1.60 \mathrm{~mW}$ in a spot size of approximately $0.7 \mathrm{~cm}^{2}$. In Fig. 3, additional data taken with a $B$-field gradient of $7.22 \mathrm{G} / \mathrm{cm}$ are shown as upward pointing triangles. Relative power in the three pairs of trapping laser beams were then varied, leaving them in the ratio of 17.2:30.2:40.7. Data taken with this substantially different balance are shown as downward pointing triangles. Finally, data were taken for repump laser powers (with fixed beam spot size) of 0.88 and $0.03 \mathrm{~mW}$. These are shown as stars for a single detuning and intensity of the trapping laser.

When plotted against the saturation parameter, most of the data taken in this experiment lie on a single well-defined curve for $s<1.25$. This implies that a simple function of the single variable $s$ can be used to estimate the excited state fraction in a MOT as long as $s<1.25$. Clearly, the data cannot be made to fit such a simple function for $s>1.25$. Perhaps this is because the two-level approximation inherent in these models is less valid as the detuning from resonance is increased. That is, when the detuning starts to become comparable to the Zeeman splitting, then the two-level model completely breaks down. One can also see that for $s>1.25$ the data seem to scatter with amplitudes that are greater than their assigned error bars. This is not due to an underestimation of uncertainty in measured excited fraction because the data are reproducible to within the error bars. Rather, the apparent scatter is due to $s$ ceasing to be a good parameter. Once again, this is thought to be due to a breakdown in the simple two-level models. It should be noted that values of $s$ beyond about 1.25 correspond to a region of MOT parameter space that is rarely frequented in experiments due the extremely low number of trapped atoms that result.

Curves from the one-parameter and two-parameter models discussed above, fit to the data for $s<1.25$, are indicated by solid and dashed lines, respectively. Both fit the data extremely well over this range. The three-parameter model was found to be less satisfactory because the data show no indication of separate "high $s$ " and "low $s$ " regimes. Therefore, fitted values of either $\alpha$ or $\beta$ tended toward infinity, and $s_{r}$ tended to be indeterminate. To within experimental scatter, nearly all the data lie on the "universal curves" of the oneand two-parameter fits, for regions of $s$ that are of interest, even though the MOT fluorescence, which is proportional to the total number of excited atoms in the MOT, varied from very bright to barely visible when viewed with a chargecoupled device (CCD) camera. Since temperature and density are functions of trapping laser detuning and intensity, this implies that the models are valid over a wide range of MOT sizes, temperatures and densities, at least for $s<1.25$.

Histograms of the residuals from the one- and twoparameter fits are shown in Figs. 4(a) and 4(b), respectively. These histograms emphasize what is not obvious in the scatter plot of Fig. 3, namely that there are many overlapping data points, the great preponderance of which lie right on a single curve. In comparing the two models, one should note that while the two-parameter model does fit the data slightly better than the one-parameter model, the residuals for the two models are very similar over the useful region of $s$. Thus, there seems to be no real advantage of going beyond the one-parameter model. The best fit to this model yields a value of effective $I_{s}$ of $9.2 \mathrm{~mW} / \mathrm{cm}^{2}$, very close to the model-dependent result [3] by Dinneen et al. of $9.1 \mathrm{~mW} / \mathrm{cm}^{2}$. This is remarkable because the assumption by Dinneen $e t$ al. that the excited fraction would saturate at a value of 0.5 is clearly seen in Fig. 3 not to be valid. A best fit of the two-parameter model to the data gives values $C_{1}$ $=0.610$ and $C_{2}=0.645$. 

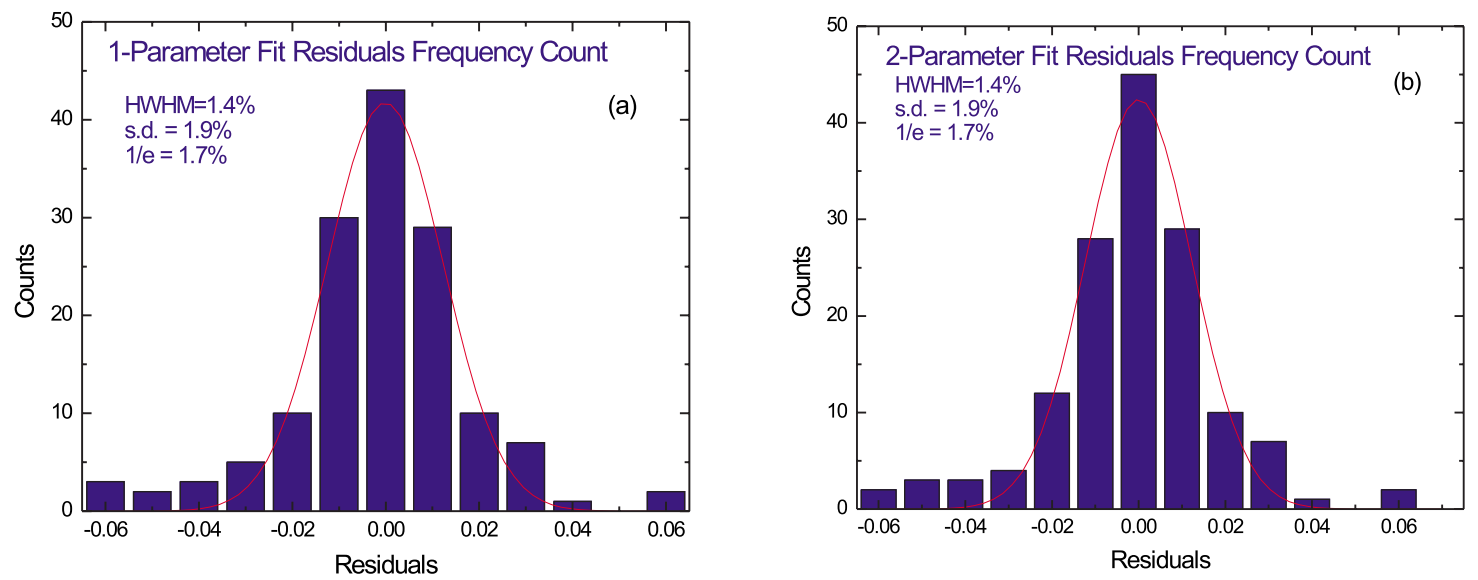

FIG. 4. (Color online) Histogram of the residuals of the one-parameter fit to the data. Only data for $s$ lying between 0 and 1.25 were used.

Thus far we have demonstrated that the simple oneparameter model of Eq. (1) does a very good job in describing the measured excited fraction in a MOT run under a wide variety of trapping conditions. We have also given a best-fit determination of $I_{s}$ to be used with Eq. (1). We now give an estimate of the uncertainty associated with that value of $I_{s}$.

Upon inverting Eq. (1) and solving for $I_{s}$, we see that $I_{s}$ $=I_{s}\left(f_{m}, I, \delta\right)$. (We use $f_{m}$ instead of $f$ to emphasize that $I_{s}$ was determined using measured values of the excited fraction.) Using standard error analysis methods, the error in $I_{s}$ can be expressed as

$$
\begin{aligned}
\frac{\Delta I_{s}}{I_{s}}= & \left\{\left(\frac{1}{1-2 f_{m}}\right)^{2}\left(\frac{\Delta f_{m}}{f_{m}}\right)^{2}+\left(\frac{\Delta I}{I}\right)^{2}\right. \\
& \left.+\left(\frac{8 \delta / \Gamma}{1+(2 \delta / \Gamma)^{2}}\right)^{2}\left(\frac{\Delta \delta}{\Gamma}\right)^{2}\right\}^{1 / 2}
\end{aligned}
$$

where $I$ refers to the total intensity of the trapping lasers at the location of the MOT.

As indicated in Eq. (12), each value of $f_{m}$ carries with it its own uncertainty. However, the range of values of $f_{m}$ and $\Delta f_{m}$ was not so great as to lead to a significant variation in $\Delta I_{s} / I_{s}$ from Eq. (13). The recommended value, then, of $I_{s}$ to be used with Eq. (1) was determined to be $I_{s}$ $=9.2 \pm 1.7 \mathrm{~mW} / \mathrm{cm}^{2}$. This allows us to make a simple estimate of the accuracy one should expect using a combination of this value of $I_{s}$ and Eq. (1). Assuming one had perfect knowledge of the trapping laser intensity and detuning, the only uncertainty in deducing the excited fraction would be $I_{s}$.
Equation (13) can then be inverted to find $\Delta f$. For example, for a typical value of $f=0.15$,

$$
\Delta f=\frac{f(1-2 f) \Delta I_{s}}{I_{s}}=\frac{(0.15)(1-0.15)(1.7)}{(9.2)}=0.02 .
$$

We note that this value in the uncertainty of $f$ is consistent with the histograms of Fig. 4.

In summary, using the model-independent method of charge transfer, the excited state fraction in a MOT of ${ }^{87} \mathrm{Rb}$ has been measured as a function of a variety of MOT parameters. Two simple models were fit to the measured fractions and were found to be excellent predictors of the excited state fractions, given only the trapping laser total intensity and detuning. The predictions of these models are robust against changes in magnetic field gradient, balance in the six laser beam intensities, and repump laser intensity. It is therefore believed that anyone trapping ${ }^{87} \mathrm{Rb}$ on the upper cycling transition, who needs to know the fraction of atoms in the excited state to very good accuracy can use Eq. (1) with $I_{s}$ $=9.2 \pm 1.7 \mathrm{~mW} / \mathrm{cm}^{2}$.

\section{ACKNOWLEDGMENTS}

The authors would like to thank S. Gensemer for providing unpublished data from his ${ }^{87} \mathrm{Rb}$ photoionization experiments. This work was supported by the Chemical Sciences, Geosciences, and Biosciences Division, Office of Basic Energy Sciences, Office of Science, U.S. Department of Energy.
[1] W. Demtröder, Laser Spectroscopy: Basic Concepts and Instrumentation (Springer, Berlin, 2002).

[2] There are two common definitions of $I_{S}$ in the literature, differing from each other by a factor of 2 . We choose to use the definition of Demtröder. Equation (1) is consistent with this definition.

[3] T. P. Dinneen, C. D. Wallace, K. Y. N. Tan, and P. L. Gould,
Opt. Lett. 17, 1706 (1992).

[4] C. G. Townsend, N. H. Edwards, C. J. Cooper, K. P. Zetie, C. J. Foot, A. M. Steane, P. Szriftgiser, H. Perrin, and J. Dalibard, Phys. Rev. A 52, 1423 (1995).

[5] J. Javanainen, J. Opt. Soc. Am. B 10, 572 (1993).

[6] X. Fléchard, H. Nguyen, R. Brédy, S. R. Lundeen, M. Stauffer, H. A. Camp, C. W. Fehrenbach, and B. D. DePaola, Phys. Rev. 
Lett. 91, 243005 (2003).

[7] R. Brédy, H. Nguyen, H. A. Camp, X. Fléchard, and B. D. DePaola, Nucl. Instrum. Methods Phys. Res. B 205, 191 (2003).

[8] H. Nguyen, X. Fléchard, R. Brédy, H. A. Camp, and B. D. DePaola, Rev. Sci. Instrum. 75, 2638 (2004).

[9] T. G. Lee, H. Nguyen, X. Fléchard, B. D. DePaola, and C. D. Lin, Phys. Rev. A 66, 042701 (2002).

[10] R. A. Valenzuela, L. J. Cimini, R. W. Wilson, K. C. Reich- mann, and A. Grot, Electron. Lett. 24, 725 (1988).

[11] Toptica, model TA100, tapered amplifier.

[12] G. Veshapidze, M. L. Trachy, M. H. Shah, and B. D. DePaola, Appl. Opt. 45, 8197 (2006).

[13] Using the "standard" definition of $I_{s}$ in the definition of $s$ on the horizontal axis of Fig. 3 causes no difficulty. It merely stretches or shrinks the horizontal scale with no effect at all on the fits. 